\title{
Clarification of the ripple phase of lecithin bilayers using fully hydrated, aligned samples
}

\author{
J. Katsaras, ${ }^{1}$ S. Tristram-Nagle, ${ }^{2}$ Y. Liu,${ }^{3}$ R. L. Headrick, ${ }^{4}$ E. Fontes, ${ }^{4}$ P. C. Mason, ${ }^{1}$ and J. F. Nagle ${ }^{2,3}$ \\ ${ }^{1}$ National Research Council, Building 459, Station 18, Chalk River Laboratories, Chalk River, Ontario, Canada K0J $1 P 0$ \\ ${ }^{2}$ Department of Biological Sciences, Carnegie Mellon University, Pittsburgh, Pennsylvania 15213 \\ ${ }^{3}$ Department of Physics, Carnegie Mellon University, Pittsburgh, Pennsylvania 15213 \\ ${ }^{4}$ Cornell High Energy Synchrotron Source, Ithaca, New York 14853
}

(Received 3 December 1999)

\begin{abstract}
Aligned samples of lipid bilayers have been fully hydrated from water vapor in a different type of x-ray chamber. Our use of aligned samples resolves issues concerning the ripple phase that were ambiguous from previous powder studies. In particular, our x-ray diffraction data conclusively demonstrate that, on cooling from the $L_{\alpha}$ to the $P_{\beta^{\prime}}$ phase, both chiral and racemic samples of dipalmitoyl phosphatidylcholine (DPPC) exhibit phase coexistence of long and short ripples with a ripple wavelength ratio $\lambda_{L} / \lambda_{S} \sim 1.8$. Moreover, the long ripple always forms an orthorhombic unit cell $\left(\gamma_{L}=90^{\circ}\right)$, strongly supporting the possibility that these ripples are symmetric. In contrast, $\gamma_{S}$ for short ripples was consistently different from $90^{\circ}$, implying asymmetric ripples. We continue to find no evidence that chirality affects the structure of rippled bilayers. The relative thermodynamic stability of the two types of ripples was investigated and a qualitative free energy diagram is given in which the long ripple phase is metastable. Finally, we suggest a kinetic mechanism, involving loss of water, that promotes formation of the metastable long ripple phase for special thermal protocols.
\end{abstract}

PACS number(s): 87.16.-b, 87.64.Bx, 61.30.Eb

\section{INTRODUCTION}

Well hydrated lipid bilayers, which serve as prototypical biomimetic systems, often exhibit an intriguing thermodynamic phase called the ripple $\left(P_{\beta^{\prime}}\right)$ phase, especially when those lipids belong to a class commonly known as lecithins [1]. One way that the corrugated $P_{\beta^{\prime}}$ phase is formed is to cool through the main phase transition from the fluid $\left(L_{\alpha}\right)$ phase, in which the time-averaged bilayers are locally flat. After further cooling (of order $10^{\circ} \mathrm{C}$ ) these rippled bilayers enter a different flat phase, typically the gel $\left(L_{\beta^{\prime}}\right)$ [2] or the subgel $\left(L_{c^{\prime}}\right)$ phase [3]. Explaining this sequence of phases has attracted numerous theoretical contributions [4-15], but progress has been impeded by uncertainty regarding the actual structure of the ripple phase.

Evidence that the $P_{\beta^{\prime}}$ phase is indeed rippled and not flat has accumulated over 25 years from freeze-fracture electron microscopy (FFEM) [16-21] and x-ray diffraction [2,2231]. The most precise structure of the $P_{\beta^{\prime}}$ phase has been obtained for the lipid dimyristoyl phosphatidylcholine (DMPC) [32] using a synchrotron data set taken at $T$ $=18^{\circ} \mathrm{C}$ and $25 \mathrm{wt}$. $\%$ water [23] [corresponding to a relative humidity (RH) $98 \%$ ]. This DMPC ripple profile has an asymmetric, sawtooth shape, rather than a smooth symmetric or simple sinusoidal shape (see Fig. 1). The long side of the asymmetric ripple is called the major or M side and the short side is the minor or $\mathrm{m}$ side. The sawtooth ripple is then described as the Mm pattern. The electron density profiles show that $\mathrm{M}$ has the same thickness as gel phase DMPC bilayers whereas the $\mathrm{m}$ side of the rippled bilayer is thinner [32]. Thus at $\sim 98 \% \mathrm{RH}$ the lattice constants for $P_{\beta^{\prime}}$ l-DMPC [33] bilayers are the following: monoclinic angle $\gamma=98.4^{\circ}$, ripple repeat $\lambda=141.7 \AA$, and bilayer repeat $d$ $=57.94 \AA$ [23]. However, even with this degree of detail the molecular packing of the lipid molecules in the Mm ripple pattern is still a matter of discussion [34].

Although there is general agreement that the asymmetric ripple pattern exists, it has also become clear that there is an additional ripple pattern, as imaged by FFEM $[16-19,21]$ and deduced from powder diffraction [28,29]. After considerable uncertainty lasting over a decade regarding the conditions for seeing different ripple patterns, it was noted by Zasadzinski [19] and then emphasized by Tenchov et al. [35]

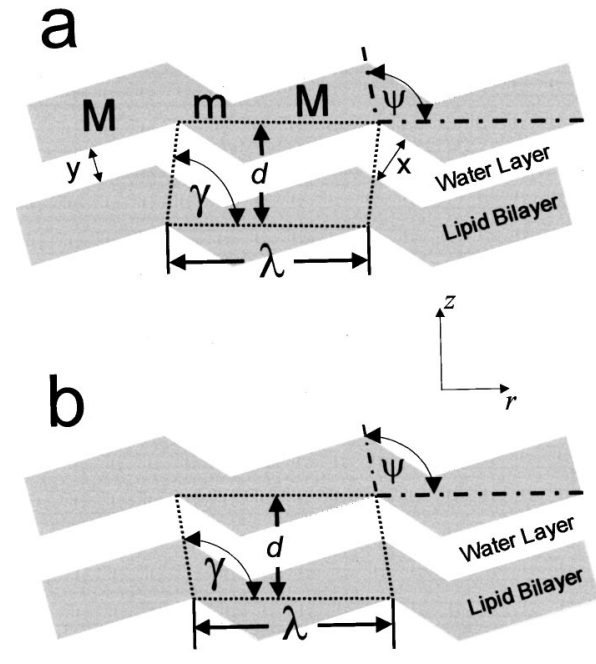

FIG. 1. Schematic drawing showing two MmMm asymmetric rippled bilayers (in a stack of many bilayers), separated by a layer of water. The unit cell, shown by the dotted parallelogram, has dimensions $d, \lambda$, and a monoclinic angle $\gamma$. In (a) $\gamma<90^{\circ}$ and in (b) $\gamma>90^{\circ}$. The water spacing between the minor sides (m) of neighboring bilayers is depicted by the variable $x$ and the water spacing between major sides (M) is depicted by the variable $y . x / y$ will vary as a function of $\gamma . \psi$ is a structural angle made between the two adjacent "kinks" in the bilayer and the horizontal. 
that the $P_{\beta^{\prime}}$ phase can differ, at least in the case of the benchmark lipid dipalmitoyl phosphatidylcholine (DPPC), depending on whether it is formed on cooling from the $L_{\alpha}$ phase or on heating from the $L_{\beta^{\prime}}$ phase. The consensus for DPPC bilayers is that the ripple phase formed upon heating from the gel phase consists of asymmetric ripples of the type described above for DMPC. We will call these short ripples and designate their properties with the subscript $S$, e.g., wavelength $\lambda_{S} \sim 140 \AA$. Our data in this paper conclusively confirm formation of this short ripple phase for DPPC upon heating.

From 1990 until recently there was a consensus that the ripple phase of DPPC formed upon cooling was a mixture consisting of a long ripple phase coexisting with the usual short ripple phase $[28,29]$. However, coexistence over an extended range of temperature generally violates the Gibbs phase rule. Coexistence was recently disputed when previously key supporting x-ray data [28] were reanalyzed (along with powder neutron diffraction data) with the conclusion that the powder diffraction data were consistent with a pure long ripple phase [36]. In the present report we decisively resolve this disagreement in favor of phase coexistence of short and long ripples. Furthermore, even among researchers who reported a long ripple phase in DPPC, there were major unresolved differences regarding the lattice parameters describing the long ripple (e.g., [28-30]).

Why then were we able to resolve this issue of bimodal ripple distribution when others could not? The reason that our data unambiguously answer many of the outstanding questions directly relates to the geometry of the sample. Previous diffraction studies used unoriented (powder) samples of multilamellar vesicles. Powder diffraction patterns reveal only the absolute $q$ values and indexing these to the twodimensional ripple unit cell can be ambiguous, especially with regard to samples consisting of mixtures of long and short ripples. In contrast, our samples are aligned, so the different diffraction spots are clearly indexed in reciprocal space. Of course, electron microscopy suffers different ambiguities due to quick freezing and irregularities in the ensuing sample, and reports using FFEM have also been conflicting in the sense that the bimodal distribution of ripples in DPPC is not consistently observed when the $P_{\beta^{\prime}}$ phase is formed on cooling (e.g., Refs. [19,21]).

Despite the many advantages offered by aligned samples, they have suffered from the fact that they apparently could not be fully hydrated from water vapor. When observed by $\mathrm{x}$-ray diffraction, aligned samples historically exhibited lamellar repeat spacings $d$ consistently smaller than in their unaligned liposomal counterparts immersed in an excess of water [31,37-40]. Since the chemical potential of water vapor in equilibrium with bulk water (100\% relative humidity) is thermodynamically the same as that of liquid water, this inconsistency came to be known as the "vapor pressure paradox" $[41,42]$. It was only in 1998 , using a newly developed sample chamber for neutron diffraction [43,44], that it was experimentally demonstrated that aligned bilayers hydrated from water vapor at $100 \%$ relative humidity have the same $d$ as their unaligned liposomal counterparts in all phases. It should be mentioned that earlier studies using a supersaturated vapor had achieved the same result for the gel phase $[45,46]$. These developments have encouraged us to build a sample chamber suitable for x-ray diffraction studies that also succeeds in fully hydrating aligned samples from water vapor. This is valuable for studying aligned rippled bilayers because the lattice constants describing the ripple ( $\lambda, \gamma$, and $d$ as shown in Fig. 1) vary as a function of water concentration $[2,23]$.

In Sec. III of this paper we report x-ray diffraction data which show that only short ripples form upon heating DPPC bilayers from the $L_{\beta^{\prime}}$ phase and that these ripples are asymmetric, in agreement with previous experimental results. Our results also show that $\gamma_{S}-90^{\circ}$ goes from positive to negative with increasing hydration (larger $d_{s}$ ) and this result is consistent with an earlier result regarding the little considered structural angle $\psi$ [32] (Fig. 1). In addition, relating molecular chirality to ripple symmetry has been an interesting theoretical issue $[13,14]$. By comparing results from $l$-DPPC and $d l$-DPPC [33] it appears that chirality plays no role in ripple phase structure, as will be elaborated in Secs. III and IV.

In Sec. IV we present data which conclusively demonstrate that DPPC multibilayers, upon cooling from the $L_{\alpha}$ phase, form two distinct phase-separated populations of ripples-a long ripple phase and a short ripple phase. For the long ripple phase the data clearly demonstrate that the twodimensional unit cell angle $\gamma=90^{\circ}$ within experimental error of $\pm 1^{\circ}$. This result is consistent with the long ripple being symmetric. Finally, in Sec. IV we note that there is strong evidence for stacking disorder for both the short and long ripple phases.

In Sec. $\mathrm{V}$ we confirm that the proportion $R$ of short to long ripple phases is very sensitive to the thermal history, as emphasized by Hatta and co-workers [28,29]. We report some variations to their thermal protocols that produced a variety of $R$ values. We also address the question of which ripple form is most thermodynamically stable and we provide support for the previous conclusion that the short ripple phase is more stable, at least near $T_{M}$. The interplay between thermodynamic stability and the kinetics of phase transformation into the two different phases is discussed.

\section{EXPERIMENTAL METHODS}

\section{A. Sample preparation}

1,2-dipalmitoyl-sn-glycero-3-phosphocholine ( $l$-DPPC) and 1,2-dipalmitoyl-rac-glycero-3-phosphocholine $(d l$-DPPC) were purchased from Avanti Polar Lipids (Alabaster, AL) and Sigma Chemical Co. (St Louis, MO), respectively, and used without further purification.

Aligned multibilayers of $l$-DPPC and $d l$-DPPC studied at the Cornell High Energy Synchrotron Source (CHESS) were prepared as follows: a concentrated lipid/methanol $(\sim 10 \mathrm{mg} / \mathrm{ml})$ solution was deposited on the outer surface of a clean cylindrical glass substrate (radius $\sim 0.0175 \mathrm{~m}$ ) using an Iwata HP-A airbrush (Anest Iwata Corp., Japan). After deposition the sample was placed under vacuum for a period of $24 \mathrm{~h}$ (to remove any traces of solvent) and subsequently annealed for an additional $24 \mathrm{~h}$ at a temperature of $60^{\circ} \mathrm{C}$ in a bell jar whose atmosphere was saturated with water vapor. The resultant aligned multibilayer stacks contained roughly 2000 bilayers. The samples studied at Carnegie Mellon University $(\mathrm{CMU})$ were prepared in a similar way except that a 


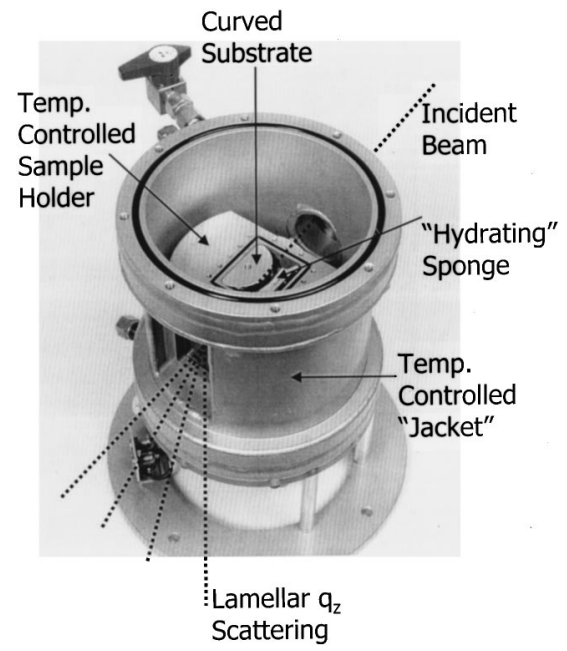

FIG. 2. Photograph of the x-ray sample chamber. The inner sample holder is a sealed unit (shown with the sealing panel removed) and contains the cylindrical substrate and the hydrating sponge. The sample holder is thermally isolated from the outer jacket. Only two of the four aluminized Mylar windows in the outer jacket can be seen.

modified "rock and roll" method [46] was used instead of an airbrush and long annealing at high temperatures.

\section{B. Sample chamber}

The sample chamber shown in Fig. 2 was designed and constructed at the Chalk River Laboratories. The design was based on two principles [44]. First, temperature gradients were minimized by the standard practice of using a massive jacket to enclose an inner sample holder. The temperatures of the jacket and the sample holder, both made of aluminum, were individually controlled by water circulating from two temperature controlled baths. This design feature allowed the temperature of the jacket to be slightly higher than the temperature of the sample holder in order to minimize water condensation on the low mass sample holder windows. Aluminized Mylar windows were used in both the jacket and the sample holder. The second principle [47] was to increase the effective evaporative surface of the pure water that interfaces with the vapor phase by using a porous sponge close to the lipid film in addition to pools of excess water not in direct contact with the lipid film. This chamber has succeeded in producing the same $d$ for aligned samples as for unoriented samples in excess water, even for the challenging $L_{\alpha}$ phase [44]. Initial hydration was accomplished in the $L_{\alpha}$ phase and required only a few hours.

\section{X-ray diffraction}

The axis of the cylindrical sample was oriented perpendicular to the beam and also to the higher resolution scattering plane, which was the lamellar scattering plane in Fig. 2. Data at the highest resolution $\left(\delta q_{z}=0.001 \AA^{-1}, \delta q_{x}\right.$ $=0.003 \AA^{-1}$ ) were obtained at CHESS (Ithaca, NY), using the D-1 station. Monochromatic $x$ rays $(\lambda=1.3808 \AA)$ were obtained using a tungsten/silicon synthetic multilayer having a repeat spacing of $22.5 \AA$, a nominal $\Delta \lambda / \lambda$ of $1 \%$, and a mosaic of $\sim 0.25^{\circ}$. The charge coupled device (CCD) detec-

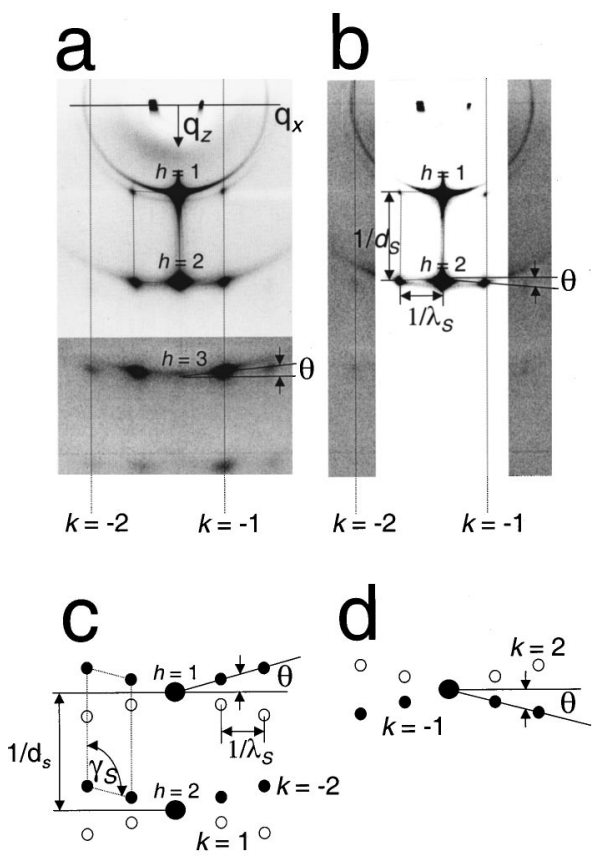

FIG. 3. Two-dimensional diffraction patterns at $39 \pm 1{ }^{\circ} \mathrm{C}$ of (a) $l$-DPPC and (b) $d l$-DPPC ripple phases formed on heating from the $L_{\beta^{\prime}}$ phase. The lattice parameters for these two samples are given in lines 1 and 3 of Table I, respectively. The diffraction patterns illustrate how the lattice parameters $(d, \gamma$, and $\lambda)$ are obtainable from the raw data. (c) and (d) are schematics of the diffraction patterns corresponding to (a) and (b), respectively, and are used to better illustrate the "smile" (c), $\gamma>90^{\circ}$, and the "frown" (d), $\gamma<90^{\circ}$. The most intense reflections arising from the ripple are denoted by solid circles while those that are less intense are depicted by open circles. The different background levels within the diffraction patterns in (a) and (b) give the impression that they are a composite of two exposure times. This is not the case; instead they are due to use of a different contrast to show both strong and weak reflections. The sample-to-detector distance was $0.581 \mathrm{~m}$.

tor, built by Tate et al. [48], has a $1024^{2}$ array of pixels, each of active size $50 \mu \mathrm{m}^{2}$. The distance from the sample to the detector was varied from $12.7 \mathrm{~cm}$ to $58.1 \mathrm{~cm}$. The flux at the sample was of the order of $10^{12}$ photons $\mathrm{mm}^{-2} \mathrm{~s}^{-1}$. Exposures to the synchrotron beam were between 0.1 and $15 \mathrm{~s}$.

Data for long equilibration times and under numerous temperature and humidity protocols were obtained at CMU using the Chalk River sample chamber and a Rigaku H3R rotating anode operated at $5.2 \mathrm{~kW}$ with a PhosphorImager detector (Molecular Dynamics, Sunnyvale, CA). X-ray exposures were $0.5 \mathrm{~h}$ to $7 \mathrm{~h}$ and resolution was about $0.04 \AA^{-1}$.

\section{III. $P_{\beta^{\prime}}$ PHASE ON HEATING}

Figures 3(a) and 3(b) show two-dimensional diffraction patterns for rippled phase $l$-DPPC and $d l$-DPPC multibilayers, respectively, formed upon heating the $L_{\beta^{\prime}}$ phase to $39^{\circ} \mathrm{C}$. These diffraction patterns show that the $P_{\beta^{\prime}}$ phase for both $l$ - and $d l$-DPPC bilayers consist of only one population of ripples. The unit cell parameters for this short $(S)$ ripple phase are defined in Fig. 1 and will be called $d_{S}, \lambda_{S}$, and $\gamma_{S}$. Values for the data shown in Fig. 3 are given in Table I. Table I also shows previous results, obtained predominantly for fully hydrated $P_{\beta^{\prime}}$ phase $l$-DPPC bilayers formed on 
TABLE I. Structural parameters of fully hydrated $P_{\beta^{\prime}}$ DPPC bilayers at a temperature of $39^{\circ} \mathrm{C}$ formed on heating from the $L_{\beta^{\prime}}$ phase.

\begin{tabular}{lccc}
\hline \hline $\begin{array}{l}d_{S} \\
(\AA)\end{array}$ & $\begin{array}{c}\lambda_{S} \\
(\AA)\end{array}$ & $\begin{array}{c}\gamma_{S} \\
(\mathrm{deg})\end{array}$ & Reference \\
\hline 68.8 & 141.9 & 92 & Present $^{\mathrm{a}, \mathrm{b}}$ \\
72.3 & 154 & 88 & Present $^{\mathrm{a}}$ \\
70.6 & 145.8 & 88 & Present $^{\mathrm{c}}$ \\
70.6 & 140.0 & 92.9 & {$[28]^{\mathrm{a}}$} \\
72.3 & 134.3 & 91.4 & {$[29]^{\mathrm{a}}$} \\
72.1 & 136.8 & 95.3 & {$[30]^{\mathrm{a}}$} \\
71 & 200 & 90 & {$[24]^{\mathrm{c}}$} \\
\hline \hline
\end{tabular}

${ }^{\mathrm{a}} l$-DPPC.

${ }^{\mathrm{b}}$ Sample is not fully hydrated.

${ }^{\mathrm{c}} d l$-DPPC.

heating. Agreement is generally satisfactory for $d_{S}$ and $\lambda_{S}$ except for the results of Hentschel and Rustichelli [24]. A single population of ripples has been deduced from a number of other diffraction studies of DPPC, but in some cases it was not clear either that the $P_{\beta^{\prime}}$ phase was formed on heating $[2,24]$ and/or that the samples were fully hydrated $[23,26,27]$. It should be noted that all three lattice parameters $\left(d_{S}, \lambda_{S}\right.$, and $\left.\gamma_{S}\right)$ (Fig. 1) vary as a function of hydration $[2,23]$, as is indicated by the difference in our two results for $l$-DPPC shown in Table I.

The unit cell angle $\gamma$ is often used as an indicator of the symmetry of the ripples [13,31]. Figures $1(\mathrm{a})$ and $1(\mathrm{~b})$ show unit cells for asymmetric ripples having $\gamma<90^{\circ}$ and $\gamma$ $>90^{\circ}$, respectively. Either of these unit cells is allowed for asymmetric ripples (and indeed, both are seen in our data) because the water spacing between the minor $\mathrm{m}$ sides of neighboring bilayers $(x)$ and that between major $\mathrm{M}$ sides $(y)$ are independent variables that depend upon details of the interactions that change with conditions, such as temperature, water content, and lipid specs. As the difference $x-y$ varies, $\gamma$ must vary. Clearly, $\gamma=90^{\circ}$ is also allowed for asymmetric ripples, so $\gamma=90^{\circ}$ does not necessarily confirm the presence of symmetric ripples [49]. On the other hand, if the ripples are symmetric, i.e., the major and minor sides are indistinguishable (Fig. 4), and if the interbilayer interactions have a single minimum, as is usually the case, then $\gamma=90^{\circ}$ is normally required by symmetry [50].

The method of measuring $\gamma$ is indicated in Fig. 3. The lamellar index is $h$ and the ripple index is $k$, as indicated in Figs. 3(a) and 3(b). For a single domain of ripples, the layer lines, given by $\mathbf{q}_{h k}=h \mathbf{A}+k \mathbf{B}$ for fixed $h$ and varying $k$, are

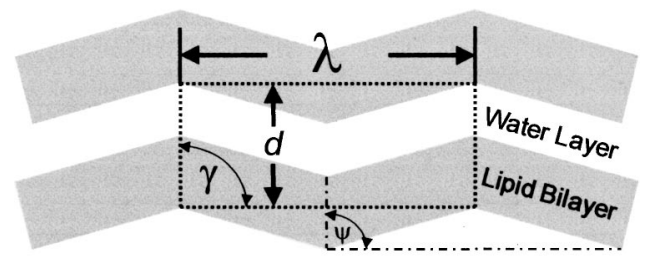

FIG. 4. Schematic drawing of a symmetric ripple whereby $M$ and $\mathrm{m}$ are indistiguishable. For this type of bilayer a $\gamma$ of $90^{\circ}$ would be expected. Moreover, $\gamma=\psi$.

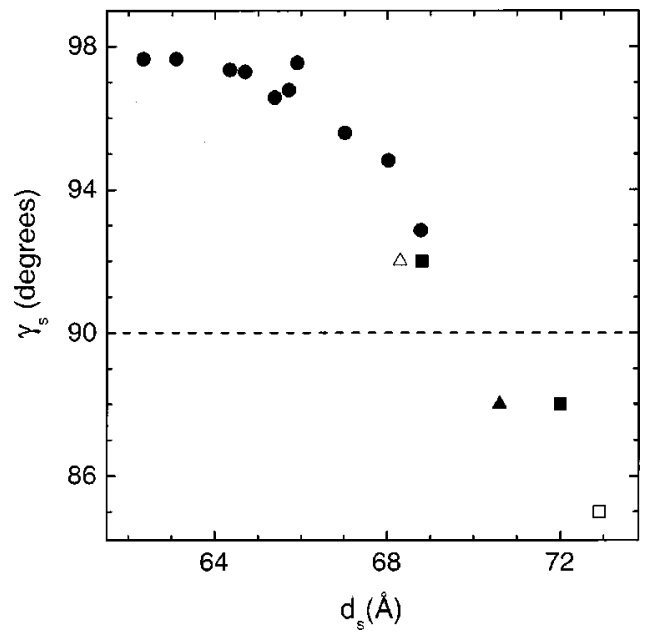

FIG. 5. Unit cell angle $\gamma_{S}$ versus $d_{S}$. Solid symbols are for rippled bilayers formed upon heating while open symbols represent short rippled bilayers formed upon cooling. All data are for $l$-DPPC except for the triangles which are for $d l$-DPPC. The solid circles are data taken from Ref. [23].

tilted by angle $\gamma=90^{\circ}+\theta$ from the $q_{z}$ axis because $\mathbf{B}$ is perpendicular to the real space unit cell vector tilted by angle $\gamma$ from the horizontal. Layer lines with both angles $90^{\circ} \pm \theta$ necessarily appear in the data because the sample consists of an in-plane powder. As was emphasized by Sun et al. (see Fig. 1 in Ref. [32]), the symmetry breaking of the Mm asymmetric ripple requires that the peaks with small negative values of $k$ be more intense than those with small positive values of $k$. This pattern is indicated by the schematic drawings [Figs. 3(c) and 3(d)]. For the data in Figs. 3(a) and 3(b), the weaker $(h, k)$ peak is not distinguishable from the stronger $(h,-k)$ peak because of the disorder and mosaic spread that accompanies high hydration, and the angle $\theta$ is small. However, the position of a single apparent peak for small nonzero $k$ is closer to the $(h,-k)$ peak, so a "smiling", layer line, as in Fig. 3(a), indicates $\gamma>90^{\circ}$, whereas a "frowning", layer line, as in Fig. 3(b), indicates $\gamma<90^{\circ}$. It should also be noted that, for some of our data, the $(h, k)$ and $(h,-k)$ peaks can both be seen when $k$ is larger because these peaks are better separated in the $q_{z}$ direction. Also, analysis shows that the ratio of $(h, k)$ to $(h,-k)$ intensity increases for higher $k$ 's [32].

The first two lines in Table I show that $\gamma_{S}-90^{\circ}$ changes sign as $d_{S}$ increases for $l$-DPPC. Wack and Webb [23] found that $\gamma_{S}$ decreased as $d_{S}$ increased, as shown in Fig. 5, but their largest $d_{S}$ was only $68.78 \AA$. In Fig. 5 we show that our results agree with those in Ref. [23] and furthermore we indicate that $\gamma_{S}-90^{\circ}$ does not decay to zero, but continues to decrease to negative values.

Let us first relate the hydration results in Fig. 5 to another structural parameter of the basic rippled bilayer introduced by Sun et al. [32], which is the angle $\psi$ shown in Fig. 1. Let us note that the ratio of bilayer thicknesses of the $\mathrm{M}$ and $\mathrm{m}$ sides increases as $\psi$ increases. Sun et al. [32] obtained $\psi$ $=99^{\circ}$ for DMPC. In the limit of complete dehydration the necessity of fitting the rippled bilayer together requires $\gamma$ to be equal to $\psi$, so the increase in $\gamma_{S}$ with dehydration can be understood as an approach to $\psi$. As more water is added, 


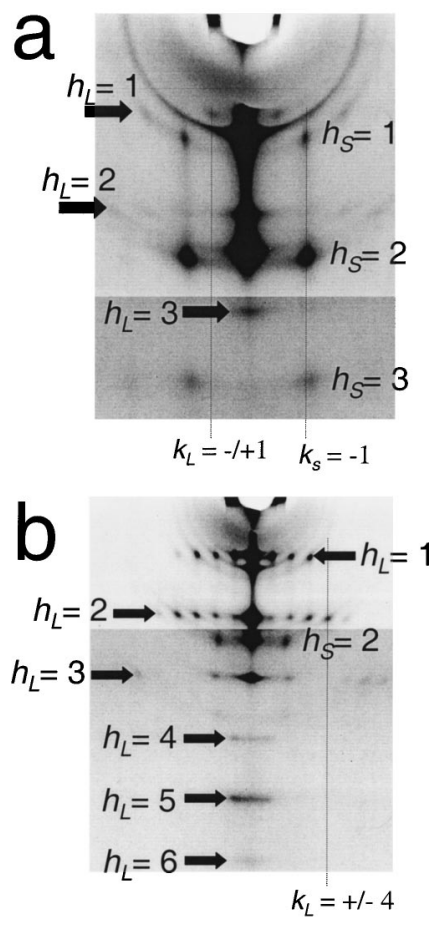

FIG. 6. Two-dimensional diffraction patterns at $39^{\circ} \mathrm{C}$ of $P_{\beta^{\prime}}$ (a) $l$-DPPC and (b) $d l$-DPPC ripple phases formed on cooling from the disordered $L_{\alpha}$ phase. The coexistence of two patterns is shown by the indices $\left(h_{S}, k_{S}\right)$ for the short ripple phase and the indices $\left(h_{L}, k_{L}\right)$ for the long ripple phase. The lattice parameters are given in lines 1 and 2, respectively, in Table II. The bold arrows identify the family of peaks due to the long ripple. As in Fig. 3, the diffraction patterns are a single exposure with different contrasts to emphasize different orders. The sample-to-detector distance was 0.581 $\mathrm{m}$ for (a) and $0.277 \mathrm{~m}$ for (b)

however, coupling between $\psi$ and $\gamma$ may be weakened and it is even possible, as indicated by our data (Fig. 5), for $\gamma$ to become less than $90^{\circ}$. Figure 1 shows schematically that this scenario increases the water spacing between the minor sides $(x)$ more than between the major sides $(y)$. This scenario is plausible since the interbilayer interactions are not expected to be the same between the different types of side.

With regard to chirality our data show that there is no significant difference between the short ripple structure of the racemate $d l$-DPPC and chiral $l$-DPPC (Table I). As mentioned previously, the effect of molecular chirality on ripple phases has been of theoretical interest $[13,14]$. A continuum Landau theory predicted that achiral bilayers should be symmetric [13], but it was subsequently shown experimentally using DMPC bilayers that achiral bilayers have $\gamma \neq 90^{\circ}$ and are therefore asymmetric [31]. The present experimental results, now in the case for DPPC, more generally support the earlier conclusion that chirality plays little role in ripple structure.

\section{IV. $P_{\beta^{\prime}}$ PHASE ON COOLING}

Figure 6 shows the diffraction patterns from fully hydrated, aligned (a) $l$-DPPC and (b) $d l$-DPPC rippled bilayers at $39^{\circ} \mathrm{C}$ formed by cooling from the $L_{\alpha}$ phase. Contrary to the suggestion of Mason et al. [36], Fig. 6 decisively confirms the interpretation of Yao et al. [28] and Matuoka et al.
TABLE II. Structural parameters of fully hydrated $P_{\beta^{\prime}}$ DPPC bilayers at a temperature of $39^{\circ} \mathrm{C}$ formed on cooling from the $L_{\alpha}$ phase.

\begin{tabular}{lcccccc}
\hline \hline $\begin{array}{l}d_{L} \\
(\AA)\end{array}$ & $\begin{array}{c}\lambda_{L} \\
(\AA)\end{array}$ & $\begin{array}{c}\gamma_{L} \\
(\mathrm{deg})\end{array}$ & $\begin{array}{c}d_{S} \\
(\AA)\end{array}$ & $\begin{array}{c}\lambda_{S} \\
(\AA)\end{array}$ & $\begin{array}{c}\gamma_{S} \\
(\mathrm{deg})\end{array}$ & Reference \\
\hline 86.6 & 258 & 90 & 72.9 & 140 & 85 & Present $^{\mathrm{A}}$ \\
82.2 & 255 & 90 & 68.3 & 144 & 92 & Present $^{\mathrm{a}}$ \\
82.2 & 266 & 90 & 70.9 & 136.4 & 95 & {$[28]$} \\
82.1 & 262.5 & 87.3 & 72.7 & 129.7 & 96.4 & {$[29]$} \\
86.2 & 261.9 & 107.3 & $72.1^{\mathrm{b}}$ & $136.8^{\mathrm{b}}$ & $95.3^{\mathrm{b}}$ & {$[30]$} \\
73 & 330 & 126 & & & & {$[36]$} \\
\hline
\end{tabular}

a $d l$-DPPC.

${ }^{\mathrm{b}}$ Structural parameters determined from phase on heating.

[29] that there are two distinct populations of ripples each exhibiting different $d, \lambda$, and $\gamma$ given in Table II. To the best of our knowledge, all previous diffraction studies of ripple phase bilayers formed on cooling from the $L_{\alpha}$ phase were carried out using unaligned, powder preparations of DPPC [28-30,36]. As such, previous conclusions were based on fitting two-dimensional (2D) Miller indices $(h, k)$ to $1 \mathrm{D}$ diffraction data. This approach, although notably successful for obtaining electron density profiles when a single phase is present [32], has clearly led to conflicting conclusions when more than one ripple phase is present (e.g., $[28,30,36])$. In contrast, the use of aligned multibilayer stacks provides clear indexing and differentiation of the two different ripple phases as seen in Fig. 6. It is then unambiguous to obtain all six lattice parameters $\left(d_{S}, d_{L}, \lambda_{S}, \lambda_{L}, \gamma_{S}\right.$, and $\left.\gamma_{L}\right)$ for both long and short ripple phases directly from the twodimensional diffraction pattern.

Results are given in Table II for the coexisting short and long ripple phases. Table II shows satisfactory agreement of our results for $d_{S}, d_{L}, \lambda_{S}$, and $\lambda_{L}$ with those studies that found two ripple populations. However, there is considerable apparent disagreement for the $\gamma$ angles, and this is the parameter that is most important when considering ripple symmetry. All studies agree that $\gamma_{S}-90^{\circ}$ is nonzero. The difference in our sign for $\gamma_{S}-90^{\circ}$ for $l$-DPPC compared to others may be simply due to using the complementary definition of the $\gamma$ angle. This distinction was first made by Sun et al. [32]. The major difference in Table II is for the $\gamma_{L}$ values. We have found repeatedly for both $l$-DPPC [Fig. 6(a)] and $d l$-DPPC [Fig. 6(b)], as well as for myristoyl-palmitoyl phosphatidylcholine (MPPC) [3], that $\gamma_{L}=90^{\circ}$. Only Yao et al. [28] reported this, but the same group [29] later reported $\gamma_{L} \neq 90^{\circ}$. Rappolt and Rapp [30] stated that their long ripple was symmetric, which seems inconsistent, as discussed earlier, with their $\gamma_{L} \neq 90^{\circ}$. Compared to the present results from aligned samples, the earlier data obtained from powder samples were generally less likely to enable one to decide whether $\gamma=90^{\circ}$. We emphasize that the result $\gamma_{L}$ $=90^{\circ}$ is quite robust in two ways. First, for any value of $h$ there were visible peaks for several values of $k$ [Fig. 6(b)] so $\gamma_{L}$ could be determined more reliably than $\gamma_{S}$. Second, we have yet to see any evidence for $\gamma_{L} \neq 90^{\circ}$ for any lipid, $l$-DPPC, $d l$-DPPC, or the mixed chain lipid MPPC [3], at any temperature or humidity.

So what is the structure of the long ripple? Our present 


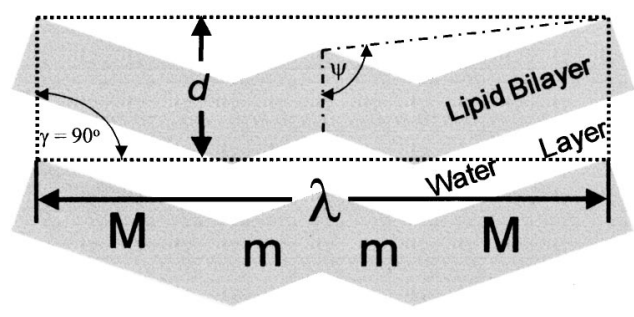

FIG. 7. Schematic drawing of the symmetric, W-shaped ripple having a repeat of $\mathrm{MmmM}$ or mMMm. As was the case of the symmetric ripple in Fig. 4 a W-shaped ripple $\gamma$ of $90^{\circ}$ is highly probable. However, unlike the symmetric ripple, $\gamma \neq \psi$.

data strongly suggest that it is symmetric because $\gamma_{L}=90^{\circ}$ for all our long ripple phases. Since $\gamma=90^{\circ}$ is only accidental for asymmetric ripples but required for symmetric ripples, we think it most unlikely that asymmetric long ripples would always have $\gamma_{L}=90^{\circ}$. In contrast, the short ripple phase usually has $\gamma_{S} \neq 90^{\circ}$ and must therefore have asymmetric ripples. Electron microscopy suggests a $\mathrm{W}$-shaped ripple that is essentially formed by the attachment of two short Mm ripples back to back to form an MmmM unit cell as depicted in Fig. $7[17,21]$. This model is consistent with the result $\lambda_{L}<2 \lambda_{S}$ because the $\mathrm{Mm}$ ripple and the $\mathrm{mM}$ ripple should not be collinear in the MmmM unit cell [51], as seen in Fig. 7.

What are the prospects of using our x-ray data to obtain the long ripple profile to test the W-ripple proposal? Since $\gamma_{L}=90^{\circ}$, the $(h, k)$ and $(h,-k)$ peaks for in-plane powders necessarily coincide in the data. Furthermore, if the long ripple is symmetric, the $(h, k)$ peak must have the same intensity as the $(h,-k)$ peak, which would simplify analysis following the method of Sun et al. [32]. Such an analysis would look promising since there are as many as 22 independent $(h,|k|)$ peaks in some of our data (compared to 26 peaks in the DMPC data [23,32]). Unfortunately, solution of the $\mathrm{x}$-ray phase problem suffers a complication if the long ripple is the symmetric $\mathrm{W}$ ripple, because $\mathrm{W}$ ripples are not centrosymmetric. Therefore, the relative $\mathrm{x}$-ray phases for the $(h, k)$ peaks with different $h$ 's would generally be complex instead of real. This prospect is presently being pursued [52].

Finally, the gray scale for Fig. 8 has been deliberately

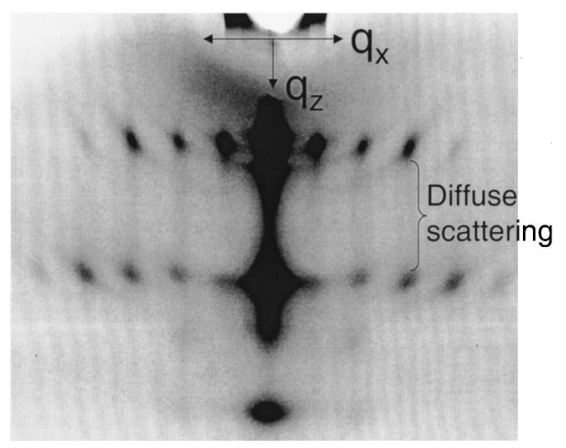

FIG. 8. Two-dimensional diffraction pattern of predominantly long rippled $d l$-DPPC bilayers formed on cooling and clearly exhibiting diffuse scattering which, most probably, corresponds to compressional disorder of the bilayer spacing $d$ along the ripple stacking $z$ direction. Exposure time $10 \mathrm{~s}$, sample-to-detector distance $0.581 \mathrm{~m}$. overexposed in order to call attention to diffuse scattering. The most prominent weak scattering in Fig. 8 is along circular arcs and is clearly due to mosaic spread, which is intrinsically uninteresting. However, there are also vertical straight lines of diffuse scattering along the $q_{z}$ direction that run between the $(h, k)$ and $(h+1, k)$ peaks, for both $k=0$ and $k \neq 0$. This phenomenon occurs for both long and short ripple phases formed upon either heating or cooling. The relative sharpness of these lines makes this diffuse scattering rather different from the far stronger diffuse scattering that we obtain from the same samples in the $L_{\alpha}$ phase and which will be the subject of another paper. This contrast suggests that disorder in the ripple phase is not like the fluctuations that occur in the Caille theory $[53,54]$ and that have been proven to describe the diffuse scattering in the $L_{\alpha}$ phase [55]. Our tentative interpretation of the ripple phase diffuse scattering is obtained by comparison to the optical transforms of disordered planar systems described by Hosemann and Bagchi [56]. Of the cases considered, our data are qualitatively most similar to Figs. 4 and 5 in Chapter 9 of Ref. [56], implying that the corresponding bilayer disorder is a result of compressional disorder (along the ripple stacking $z$ direction) rather than disorder in the shearing $r$ direction of the bilayer (Fig. 1).

\section{METASTABILITY AND THERMAL HISTORY}

The coexistence of two separate phases over the nonzero temperature range encompassing the ripple phase is not allowed in thermodynamic equilibrium by Gibbs phase rule, which states that the number of independent thermodynamic variables is $f=c-\phi+2$ where $c$ is the number of chemical components in the system and $\phi$ is the number of separate phases. For $l$-DPPC there are two components, lipid and water. The phases involved are liquid water, water vapor, and the lipid/water ripple phases. If there are two ripple phases, $\phi=4$, resulting in $f=0$. Therefore, equilibrium coexistence can occur at only one temperature (which is consistent with all the other known bilayer phase transitions [57]). This implies that for general $T$ either the long or short ripple phase is metastable. Tenchov et al. [35] argued that the short ripple phase is more stable because the enthalpy of the main transition on heating $\left(L_{\alpha} \rightarrow P_{\beta^{\prime}}\right)$ is larger than the enthalpy of the main transition on cooling $\left(P_{\beta^{\prime}} \rightarrow L_{\alpha}\right)$. However, this argument ignores the contribution of entropy to the free energy. This point was noted by Mason et al. [36] who then argued, based on low angle neutron diffraction data, that the short ripple phase is also more disordered, so it would have a lower free energy from both the enthalpic and entropic contributions. However, thermodynamic disorder may not coincide with scattering disorder. We therefore decided to study the metastability issue by annealing samples consisting of mixtures of the two phases. If the proportion of one of the phases consistently increased, then that phase would clearly be the thermodynamically stable one. In the past this strategy was used successfully to resolve similar issues regarding subgel/gel phase metastability [58,59].

We prepared a mixture of ripple phases by cooling the sample from the $L_{\alpha}$ phase. However, this led us to consider a second interesting phenomenon, namely, that the proportion of the two different ripple phases was quite variable. 
TABLE III. Low resolution diffraction results for a variety of thermal protocols using $l$-DPPC bilayers.

\begin{tabular}{lcccccc}
\hline \hline $\begin{array}{l}\text { Sample } \\
\text { (line) }\end{array}$ & $\begin{array}{c}\tau_{M}{ }^{\mathrm{a}} \\
(\mathrm{h})\end{array}$ & $\begin{array}{c}T_{i}^{\mathrm{b}} \\
\left({ }^{\circ} \mathrm{C}\right)\end{array}$ & $\begin{array}{c}\tau_{i}^{\mathrm{c}} \\
(\mathrm{h})\end{array}$ & $\begin{array}{c}R \\
\left(I_{S} / I_{L}\right)\end{array}$ & $\begin{array}{c}d_{L} \\
(\AA)\end{array}$ & $\begin{array}{c}d_{S} \\
(\AA)\end{array}$ \\
\hline A-1 & 0 & 38.5 & 4 & 20 & 85 & 73 \\
A-2 & & 34.5 & 24 & 20 & 85 & 73 \\
A-3 & & 38.5 & 4 & 20 & 83 & 72 \\
B-1 & 0 & 41 & 19 & 3.4 & 87 & 72 \\
C-1 & 5 & 39.5 & 5 & 0.1 & 88 & 73 \\
C-2 & & 39.5 & 37 & 0.1 & 85 & 73 \\
D-1 & 25 & 41 & 4 & 0 & 83 & \\
D-2 & & 37.5 & 40 & 0 & 82 & \\
D-3 & & 37.5 & 13 & 5 & 80.5 & 68 \\
D-4 & & 37.5 & 9 & $\infty$ & & 67 \\
E-1 & 22 & 41 & 2 & 0.1 & 84 & 71 \\
E-2 & & 41.4 & 3 & 0.5 & 85 & 70 \\
E-3 & & 41.4 & 16 & 1 & 85 & 69 \\
E-4 & & 41.4 & 59 & 7 & 85 & 70 \\
\hline \hline
\end{tabular}

${ }^{\mathrm{a}} \tau_{M}$ is the wait time at $T_{M} \cdot T_{M}$ is $41.4{ }^{\circ} \mathrm{C}$.

${ }^{\mathrm{b}} T_{i}$ is the quenching temperature.

${ }^{\mathrm{c}} \tau_{i}$ is the wait time at $T_{i}$, after which time data were collected.

This was first emphasized by Matuoka et al. [29], who showed that fast cooling through the main transition temperature $T_{M}=41.4^{\circ} \mathrm{C}$ resulted in predominantly a short ripple phase, with the long ripple phase becoming prominent upon slower continuous cooling. They suggested that the time spent near $T_{M}$ was the important variable. We have tested this hypothesis and several others. Results shown in Table III will now be discussed sequentially.

All samples were heated to $T=48^{\circ} \mathrm{C}$, at which point they were in the $L_{\alpha}$ phase. Sample A was cooled continuously over a two hour period to $T_{1}=38.5^{\circ} \mathrm{C}$ where it was held for time $\tau_{1}=4 \mathrm{~h}$ before taking $\mathrm{X}$-ray data, which gave the results for $R, d_{L}$, and $d_{S}$ shown in line A-1 in Table III. $R$ is the ratio of short to long ripple phase as estimated from the ratio of the maximal intensities $\left(I_{S} / I_{L}\right)$ of their respective $(1,0)$ peaks. The result $R=20$ indicates that continuous cooling through $T_{M}\left(\tau_{M}=0\right)$ results in mostly short ripple. The sample was then cooled to $T_{2}=34.5^{\circ} \mathrm{C}$ for $\tau_{2}=24 \mathrm{~h}$. Line A-2 shows that $R$ does not change upon further cooling. This result differs from that reported in Ref. [1] for a different lecithin, where the values for $R$ decreased upon cooling. Line A-3 indicates thermal reversibility, also reported by Cunningham et al. [1]. B-1, in Table III shows that the target cooling temperature $T_{1}$ for first formation of the ripple phase affects $R$, but there is still mostly short ripple $(R>1)$. The major difference for sample $\mathrm{C}$ was that the temperature was held just above $T_{M}$ for $\tau_{M}=5 \mathrm{~h}$ before dropping to $T_{1}$ $=39.5^{\circ} \mathrm{C}$. This resulted in mostly long ripple phase, which line $\mathrm{C}-2$ shows remained stable for $37 \mathrm{~h}$. At this point we decided to see if we could devise a thermal protocol to produce a pure long ripple phase in order to facilitate structural studies of this phase. Our most successful result is shown in line D-1 which employed long annealing at $T_{M}$ and a high $\operatorname{target} T_{1}$. However, we should note that the same nominal conditions did not always produce such a small value of $R$. This essentially pure long ripple phase was stable for $40 \mathrm{~h}$ as

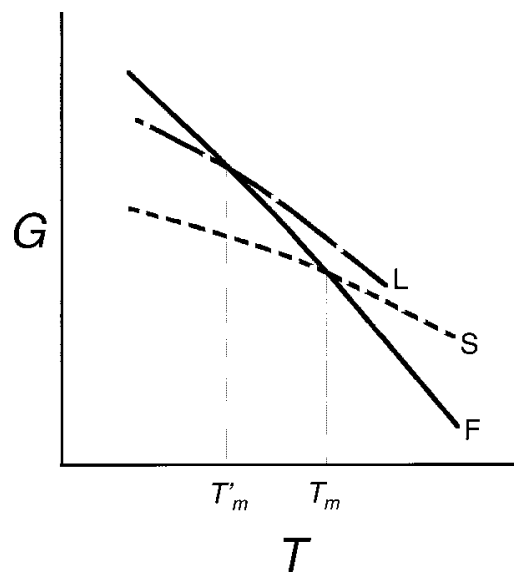

FIG. 9. Schematic diagram for free energies $G_{F}$ (fluid phase, solid curve), $G_{S}$ (short ripple phase, short dashed curve), and $G_{L}$ (long ripple phase, long dashed curve) versus temperature for fully hydrated bilayers. $T_{M}=41.4^{\circ} \mathrm{C}$.

shown in D-2. However, lines D-3 and D-4 show that the long ripple rapidly converted into the short ripple phase. Such a sigmoidal time conversion could, in principle, be due to nucleation and growth of the short ripple phase, similar to the formation of the subgel phase [59]. However, the actual explanation is that the water reservoir inadvertently dried up, so the sample became progressively dehydrated. This also caused the decrease in $d_{L}$ and $d_{S}$. This was one of the few experiments in which the long ripple converted to the short ripple. In another experiment (E-1), after obtaining mostly long ripple phase, $T_{2}$ was increased very close to $T_{M}$ and conversion of long ripple phase to short ripple phase was observed (E-2, E-3, and E-4). We found no accumulation of $L_{\alpha}$ phase during this conversion. This latter result required considerable care since the repeat spacing for the $L_{\alpha}$ phase, $d_{F}$, is close to $d_{S}$; a careful deconvolution of the intensity along a layer line showed that the loss of intensity from the long ripple phase could be accounted for by the increase in intensity of the short ripple phase. An additional useful observation (not shown in Table III) is that $R$ increased when $T_{1}$ was increased above $41{ }^{\circ} \mathrm{C}$ toward $T_{M}$.

Discussion of the preceding results is facilitated by Fig. 9, which shows a schematic of Gibbs free energies for the short ripple phase $\left(G_{S}\right)$ and the fluid $L_{\alpha}$ phase $\left(G_{F}\right)$ crossing at $T_{M}$. The question then is how should the free energy of the long ripple phase $\left(G_{L}\right)$ be placed relative to $G_{F}$ and $G_{S}$ ? The results for sample $\mathrm{E}$ in Table III imply that the long ripple phase is metastable with respect to the short ripple phase, at least when $T$ is close to $T_{M}$, so $G_{L}>G_{S}$ at $T_{M}$. The fact that long ripples form from the $L_{\alpha}$ phase at $T=41{ }^{\circ} \mathrm{C}$ requires $G_{L}<G_{F}$ at $41^{\circ} \mathrm{C}$. Therefore, in Fig. $9 G_{L}$ has been drawn to cross $G_{F}$ at an intermediate temperature that has been tentatively chosen to be $T_{M}^{\prime}=41.2^{\circ} \mathrm{C}$. This is consistent with the observation that interconversion between the two ripple phases at full hydration (the only case considered in Fig. 9) seems to occur only near $T_{M}$. Although there was no accumulation of $L_{\alpha}$ phase in our data, it is plausible that the conversion took place by first melting the long ripples to the $L_{\alpha}$ phase followed by fast conversion to the short ripple phase. This can only happen in the small $T$ interval just below $T_{M}$ where the inequality $G_{S}<G_{F}<G_{L}$ holds. Placing 
the crossing temperature $T_{M}^{\prime}$ of $G_{L}$ and $G_{F}$ close to and below $T_{M}$ is also consistent with the final observation in the preceding paragraph that $R$ increases as the initial target conversion temperature $T_{1}$ is increased above $41{ }^{\circ} \mathrm{C}$, because the long ripple phase becomes unstable relative to the fluid phase as $T$ is raised toward $T_{M}$.

We also note that it would be purely coincidental if all three curves crossed at exactly the same $T_{M}$. However, if the long ripple is the $\mathrm{W}$ ripple, formed by rearranging short ripples from $\mathrm{MmMm}$ to $\mathrm{MmmM}$ (Fig. 7), then the free energies would be expected to be nearly equal and the temperatures where $G_{S}$ and $G_{L}$ intersect with $G_{F}$ would be as proposed in Fig. 9.

Let us now consider the slope of the $G_{L}$ curve relative to the $G_{S}$ curve. As drawn in Fig. $9, G_{L}-G_{S}$ increases as $T$ decreases, so the thermodynamic driving energy $G_{F}-G_{S}$ for formation of short ripples increases faster than $G_{F}-G_{L}$ for long ripples. This is consistent with our observation that $R$ is larger when the initial incubation temperature $T_{1}$ is smaller. Since $(\partial G / \partial T)_{P}=-S$, the slope of the $G_{L}$ curve in Fig. 9 assumes that $S_{L}>S_{S}$. We now show that this agrees with the calorimetric result for the transition enthalpies [35],

$$
H_{F}-H_{L}=r\left(H_{F}-H_{S}\right),
$$

where $H_{F}$ is the enthalpy of the fluid $L_{\alpha}$ phase and the measured value of $r$ is about 0.95 [35]. Assuming that $H$ and $S$ do not change significantly for any of the phases $(F, L$, or $S)$, within the narrow temperature range $T_{M}-T_{M}^{\prime} \sim 0.2 \mathrm{~K}$, the two Clausius-Clapeyron equations for melting the two ripple phases,

$$
H_{F}-H_{L}=T_{M}^{\prime}\left(S_{F}-S_{L}\right), H_{F}-H_{S}=T_{M}\left(S_{F}-S_{S}\right),
$$

can be subtracted and Eq. (1) can be used to yield

$$
S_{L}-S_{S}=\left[\left(H_{F}-H_{S}\right) / T_{M}\right]\left[(1-r)-r\left(T_{M}-T_{M}^{\prime}\right) / T_{M}^{\prime}\right] .
$$

The last term in Eq. (3) is small compared to the first, so we conclude that $S_{L}>S_{S}$. This result disagrees with the suggestion of Mason et al. [36], which was based on small angle neutron scattering. While their result appears to be valid for large scale disorder, which is assayed by small angle scattering, we suggest that the total entropy of these phases may be dominated by disorder at the molecular scale. In any case, it is clear that calorimetry is more appropriate than scattering to obtain entropy quantitatively.

Turning back to the formation of the ripple phases, we now emphasize that free energies alone cannot explain how $R$ could be as small as 0 when $T_{1}=41^{\circ} \mathrm{C}$. Also, according to the free energy diagram in Fig. 9, there should be conversion of the long ripple phase to the short ripple phase for $T$ $<T_{M}$, but this did not occur below $T_{M}^{\prime}$ despite several attempts. To understand our experiments we must therefore include nonequilibrium kinetic considerations of ripple formation. From a kinetic point of view the nonconversion of $L$ to $S$ ripples below $T_{M}^{\prime}$ is understandable. Since the free energy for the $\mathrm{MmMm}$ and the MmmM patterns would be very similar, there is likely to be little thermodynamic driving energy. However, conversion would require concerted rearrangement of adjacent bilayers, so the energy barrier would likely be large. This argument does not apply to the temperature region between $T_{M}^{\prime}$ and $T_{M}$ because it is possible for the long ripple to convert by first melting into the $L_{\alpha}$ phase in this temperature range [60].

One consideration that we think is likely to be important for the kinetics of formation is the flow of water. It is well known that there is more water between bilayers in the fully hydrated $L_{\alpha}$ phase than in the ripple phase [2], so one kinetic process that has to occur in order to form the ripple phase from the $L_{\alpha}$ phase is removal of water. We now argue that it is plausible that there is more water in the long ripple phase than in the short ripple phase, so less water has to be removed from the long ripple phase, thereby kinetically enhancing its formation compared to formation of the short ripple phase, which is thermodynamically favored. Suppose that the long ripple consists of the same $\mathrm{M}$ and $\mathrm{m}$ sides as the short ripple. The volume of the unit cell consists of the volume of the lipid plus the volume of the water. It is safe to assume that the volume per lipid is the same in both ripple phases (for comparison the volume change in the main transition is only $3.5 \%$, e.g., Ref. [61]). Therefore, differences in unit cell volumes, suitably normalized by a factor of 2 for the smaller Mm ripple unit cell compared to the $\mathrm{MmmM}$ ripple, are due to differences in amount of water. The unit cell volumes are calculated from Tables II and III by multiplying $d, \lambda$, and an irrelevant constant length in the third dimension, with the result that the long ripple unit cell volume is 5-10\% larger than twice the short ripple unit cell volume. It should be noted that this difference is satisfactorily smaller than the difference of $39 \%$ for the unit cell volumes of the fully hydrated gel and $L_{\alpha}$ phases of DPPC [62]. Moreover, Janiak et al. [2] give equal water contents for the gel and ripple phases. Finally, why should prolonged annealing near $T_{M}$, while in the $L_{\alpha}$ phase, favor formation of the "more hydrated" long ripple phase? In $L_{\alpha}$ bilayers there is an anomalous increase in $d_{F}$ on approaching $T_{M}$ [63]. Although the mechanism for this behavior is not fully resolved, several of the possibilities require more water between the bilayers near $T_{M}$. Cooling rapidly through $T_{M}$ may not give this extra water time to enter the $L_{\alpha}$ phase, so not as much water would have to leave to form either ripple phase. This would favor formation of the short ripple phase. In contrast, full equilibration at $T_{M}$ would maximally swell the $L_{\alpha}$ phase, favoring formation of the long ripple phase when the temperature is lowered.

\section{CONCLUSIONS}

We have studied the $P_{\beta^{\prime}}$ phase, formed both on heating from the $L_{\beta^{\prime}}$ phase and on cooling from the $L_{\alpha}$ phase, using aligned, fully hydrated DPPC bilayers. Our results indicate the following.

(a) On heating, only the short ripple phase forms in both $l$ and $d l$-DPPC bilayers, whereas upon cooling the same short ripple phase coexists in varying proportions with a long ripple phase.

(b) For the short ripple phase, $\gamma_{S}$ increases with decreasing hydration and decreasing $d_{S}$. This is consistent with the bilayer structural angle $\psi$ being greater than $90^{\circ}$.

(c) The result that $\gamma_{S}$ is generally different from $90{ }^{\circ} \mathrm{C}$ implies that the short ripple has an asymmetric $\mathrm{Mm}$ pattern. 
(d) The robust value $\gamma_{L}=90^{\circ} \pm 1^{\circ}$ for the long ripple phase strongly implies a symmetric ripple, possibly of the MmmM type.

(e) Comparison of results for chiral $l$-DPPC and racemic $d l$-DPPC shows little quantitative difference and no qualitative symmetry differences.

(f) From the diffuse scattering present in the 2D diffraction patterns we tentatively suggest that there is compressional stacking disorder along the $d$ direction in both the short and long wavelength ripple phases.

(g) We confirm that the proportion $R$ of short to long wavelength ripple phases is highly dependent upon the detailed cooling protocol. On several occasions we produced a nearly pure long ripple phase, although this was not a robust outcome. We suggest that the greater loss of water required to form the short ripple phase preferentially promotes the kinetics of formation of the metastable long ripple phase.

(h) From our detailed analysis of the results of ripple melting and formation we suggest a qualitative Gibbs diagram in which the long ripple phase is metastable.

\section{ACKNOWLEDGMENTS}

NSF Grant No. DMR-9311772 supported R.L.H. and E.F. and the CHESS facility. Synchrotron beam time was provided under CHESS Proposal No. FS206 (J.K.) and P727 (J.F.N.). NIH Grant No. GM44976 supported J.F.N., S.T.N., and Y.L. P.C.M. received financial support from the Natural Sciences and Engineering Research Council.
[1] B. A. Cunningham, A.-D. Brown, D. H. Wolfe, W. P. Williams, and A. Brain, Phys. Rev. E 58, 3662 (1998).

[2] M. J. Janiak, D. M. Small, and G. G. Shipley, Biochemistry 15, 4575 (1976).

[3] S. Tristram-Nagle, Y. Isaacson, Y. Lyatskaya, Y. Liu, K. Brummond, J. Katsaras, and J. F. Nagle, Chem. Phys. Lipids 100, 101 (1999).

[4] S. Doniach, J. Chem. Phys. 70, 4587 (1979).

[5] M. Marder, H. L. Frisch, J. S. Langer, and H. M. McConnell, Proc. Natl. Acad. Sci. USA 81, 6559 (1984).

[6] M. H. Hawton and W. J. Keeler, Phys. Rev. A 33, 3333 (1986).

[7] A. Georgallas and M. J. Zuckermann, Eur. Biophys. J. 14, 53 (1986).

[8] J. M. Carlson and J. P. Sethna, Phys. Rev. A 36, 3359 (1987).

[9] R. E. Goldstein and S. Leibler, Phys. Rev. Lett. 61, 2213 (1988).

[10] R. E. Goldstein and S. Leibler, Phys. Rev. A 40, 1025 (1989).

[11] W. S. McCullough and H. L. Scott, Phys. Rev. Lett. 65, 931 (1990).

[12] K. Honda and H. Kimura, J. Phys. Soc. Jpn. 60, 1212 (1991).

[13] T. C. Lubensky and F. C. MacKintosh, Phys. Rev. Lett. 71, 1565 (1993).

[14] U. Seifert, J. Shillcock, and P. Nelson, Phys. Rev. Lett. 77, 5237 (1996).

[15] C. Misbah, J. Duplat, and B. Houchmandzadeh, Phys. Rev. Lett. 80, 4598 (1998).

[16] B. R. Copeland and H. M. McConnel, Biochim. Biophys. Acta 599, 95 (1980).

[17] E. Sackmann, D. Ruppel, and C. Gebhardt, in Springer Series in Chemical Physics, edited by W. Helfrich and G. Heppke (Springer-Verlag, Berlin, 1980).

[18] A. Hicks, M. Dinda, and M. A. Singer, Biochim. Biophys. Acta 903, 177 (1987).

[19] J. A. Zasadzinski, Biochim. Biophys. Acta 946, 235 (1988).

[20] H. W. Meyer, B. Dobner, and K. Semmler, Chem. Phys. Lipids 82, 179 (1996).

[21] J. T. Woodward and J. A. Zasadzinski, Biophys. J. 72, 964 (1997)

[22] A. Tardieu, V. Luzzati, and F. C. Reman, J. Mol. Biol. 75, 711 (1973)

[23] D. C. Wack and W. W. Webb, Phys. Rev. A 40, 2712 (1989).
[24] M. P. Hentschel and F. Rustichelli, Phys. Rev. Lett. 66, 903 (1991).

[25] M. J. Janiak, D. M. Small, and G. G. Shipley, J. Biol. Chem. 254, 6068 (1979).

[26] J. Stamatoff, B. Feuer, H. J. Guggenheim, G. Tellez, and T. Yamane, Biophys. J. 38, 217 (1982).

[27] M. R. Alecio, A. Miller, and A. Watts, Biochim. Biophys. Acta 815, 139 (1985).

[28] H. Yao, S. Matuoka, B. Tenchov, and I. Hatta, Biophys. J. 59, 252 (1991)

[29] S. Matuoka, H. Yao, S. Kato, and I. Hatta, Biophys. J. 64, 1456 (1993).

[30] M. Rappolt and G. Rapp, Eur. Biophys. J. 24, 381 (1996).

[31] J. Katsaras and V. A. Raghunathan, Phys. Rev. Lett. 74, 2022 (1995).

[32] W.-J. Sun, S. Tristram-Nagle, R. M. Suter, and J. F. Nagle, Proc. Natl. Acad. Sci. USA 93, 7008 (1996).

[33] $l$ bilayers are chiral while $d l$ bilayers are racemic and contain a 50/50 mixture of opposite enantiomers $(d$ and $l)$. $d l$ bilayers are not "achiral"' but like achiral molecules lack optical activity.

[34] K. Sengupta, V. A. Raghunathan, and J. Katsaras, Phys. Rev. E 59, 2455 (1999).

[35] B. G. Tenchov, H. Yao, and I. Hatta, Biophys. J. 56, 757 (1989).

[36] P. C. Mason, B. D. Gaulin, R. M. Epand, G. D. Wignall, and J. S. Lin, Phys. Rev. E 59, 921 (1999).

[37] J. Torbet and M. H. F. Wilkins, J. Theor. Biol. 62, 447 (1976).

[38] V. A. Raghunathan and J. Katsaras, Phys. Rev. Lett. 74, 4456 (1995).

[39] J. Katsaras, V. A. Raghunathan, E. J. Dufourc, and J. Dufourcq, Biochemistry 34, 4684 (1995).

[40] S. Tristram-Nagle, H. I. Petrache, R. M. Suter, and J. F. Nagle, Biophys. J. 74, 1421 (1998).

[41] R. P. Rand and V. A. Parsegian, Biochim. Biophys. Acta 988, 351 (1989).

[42] J. F. Nagle and J. Katsaras, Phys. Rev. E 59, 7018 (1999).

[43] J. Katsaras, Biophys. J. 73, 2924 (1997).

[44] J. Katsaras, Biophys. J. 75, 2157 (1998).

[45] J. Katsaras, D. S.-C. Yang, and R. M. Epand, Biophys. J. 63, 1170 (1992).

[46] S. Tristram-Nagle, R. Zhang, R. M. Suter, C. R. Worthington, 
W.-J. Sun, and J. F. Nagle, Biophys. J. 64, 1097 (1993).

[47] Peter Rand (private communication).

[48] M. W. Tate, E. F. Eikenberry, S. O. Barna, M. E. Wall, J. L. Lowrance, and S. M. Gruner, J. Appl. Crystallogr. 28, 196 (1995).

[49] Symmetric does not mean that the ripple is simply sinusoidal with no higher harmonics.

[50] Even if some kind of Peierl's instability developed for symmetric ripples, inducing $\gamma \neq 90^{\circ}$, the ensuing difference in interbilayer forces would likely induce inequivalency in the different sides of the ripple, which leads to asymmetry.

[51] M. L. Belaya, M. V. Feigel'man, and V. G. Levadny, J. Phys. II 1, 375 (1991).

[52] K. Sengupta and V. A. Raghunathan (private communication).

[53] A. Caillé, C. R. Seances Acad. Sci., Ser. B 274, 891 (1972).

[54] R. Zhang, R. M. Suter, and J. F. Nagle, Phys. Rev. E 50, 5047 (1994).

[55] R. Zhang, S. Tristram-Nagle, W. Sun, R. L. Headrick, T. C. Irving, R. M. Suter, and J. F. Nagle, Biophys. J. 70, 349
(1996).

[56] R. Hosemann and S. N. Bagchi, Direct Analysis of Diffraction by Matter (North-Holland, Amsterdam, 1962).

[57] J. F. Nagle, Annu. Rev. Phys. Chem. 31, 157 (1980).

[58] J. F. Nagle and D. A. Wilkinson, Biochemistry 21, 3817 (1982).

[59] C.-P. Yang and J. F. Nagle, Phys. Rev. A 37, 3993 (1988).

[60] It should be mentioned that, when the sample became slightly dehydrated, complete conversion did take place, as seen in lines D-3 and D-4 in Table III. However, this latter observation still supports the conclusion that the short ripple phase is, in general, more stable.

[61] J. F. Nagle and D. A. Wilkinson, Biophys. J. 23, 159 (1978).

[62] J. F. Nagle, R. Zhang, S. Tristram-Nagle, W. Sun, H. I. Petrache, and R. M. Suter, Biophys. J. 70, 1419 (1996).

[63] J. F. Nagle, H. I. Petrache, N. Gouliaev, S. Tristram-Nagle, Y. Liu, R. M. Suter, and K. Gawrisch, Phys. Rev. E 58, 7769 (1998). 